\title{
The Critic Liquid-Gas Phase Transition Between Liquid Nitrogen and YBCO HTS Bulks: From FEM Modeling to its Experimental Study for ZFC Levitation Devices
}

\author{
António J. Arsénio ${ }^{(1)}$, Rui Melício ${ }^{(1)}$, Carlos Cardeira ${ }^{(1)}$, and Paulo J. da Costa Branco ${ }^{(1)}$
}

\begin{abstract}
This paper presents an experimentally verified finite-element multiphysics model developed to simulate the coupled electro and thermodynamic processes (electromagnetic, thermal, and fluid-flow with liquid-gas phase transition) occurring between liquid nitrogen $\left(\mathrm{LN}_{2}\right)$ and HTS YBCO bulks. The model allows estimating: 1) the thermal time constants related to bulk HTS losing/gaining its superconducting state, and mainly 2) the $\mathbf{L N}_{2}$ liquid-to-gas flow rate due to superconductor Joule losses caused by electromagnetic induction heating of it. Model accuracy is evaluated, for example, by comparing the cooling time needed to zero-field-cool the YBCO bulk, and the quantity of $\mathrm{LN}_{2}$ vaporized as the superconductor cool. Other important issues identified were time evolution and spatial distribution of temperature in the superconductor, heat flux, nitrogen liquid flow, and also nitrogen gas concentration for specific cooling scenarios. Full-scale experimental tests in our laboratory allowed us to validate with success the model and its effectiveness predictions.
\end{abstract}

Index Terms-YBCO, superconductor bulk, liquid nitrogen, HTS, heat transfer, turbulent bubbly flow, finite-element modeling, multiphysics.

\section{INTRODUCTION}

A FULL ZFC topology is now a strong option for HTS maglev devices [1], [3], [4], maximizing levitation forces and mainly minimizing Joule losses on YBCO bulks [2], [3]. During a superconductor cooling period, a liquid nitrogen phase change occurs and thus its flow around the superconductor, releasing bubbles due to the temperature gradients, mainly in the superconductor boundaries. Hence, this flow of nitrogen gas bubbles will affect the heat flow exchange capacity between $\mathrm{YBCO}$ and $\mathrm{LN}_{2}$ that must be studied for a reliable design of any ZFC-superconducting levitation device.

Manuscript received September 18, 2017; accepted January 23, 2018. Date of publication February 2, 2018; date of current version February 28, 2018. This work was supported in part by the FCT, through IDMEC, under LAETA projects UID/EMS/50022/2013 and PTDC/EEEI-EEL/4693/2014 - HTSISTELEC. The work of A. J. Arsénio was supported by the FCT fellowship SFRH/BD/117921/2016. (Corresponding author: P. J. da Costa Branco.)

A. J. Arsénio, R. Melício, C. Cardeira are with the Instituto de Engenharia Mecânica, Instituto Superior Técnico, Universidade de Lisboa, Lisboa 1049001, Portugal.

P. J. da Costa Branco is with Departamento de Engenharia Electrotécnica e de Computadores, Instituto Superior Tecnico, Universidade de Lisboa, Lisboa 1049-001, Portugal (e-mail: pbranco@tecnico.ulisboa.pt).

Color versions of one or more of the figures in this paper are available online at http://ieeexplore.ieee.org

Digital Object Identifier 10.1109/TASC.2018.2801352
Electromagnetic heating of HTS bulks is lower with ZFC than with Field Cooling (FC), as shown in [2], [3]. Hence, ZFC levitation devices, as magnetic bearings for example [4][6], will require less energy to maintain the operating temperature of YBCO bulks below their critical temperature. In this context, adequate physical models should be taken into account to not only accurately predict the $\mathrm{LN}_{2}$ consumption rates during device operation, but also its superconductor stability.

This paper shows our recent research about the coupled thermodynamic processes (thermal and liquid-gas phase transition) that occur between liquid nitrogen $\left(\mathrm{LN}_{2}\right)$ and YBCO HTS bulks when these operate submerged in it and earlier zero-field-cooled (ZFC). The objective was to develop an accurate enough twophase finite element model of the $\mathrm{LN}_{2}$-YBCO set to preview, for different operating conditions;

- The thermal time constants related to bulk HTS losing/ gaining its superconducting state and, most importantly;

- the $\mathrm{LN}_{2}$ liquid-to-gas flow rate due to superconductor Joule losses caused by electromagnetic induction heating of it.

This research proposes and validates appropriate models of the thermodynamic processes in $\mathrm{LN}_{2}$, during the initial zero field cooling of YBCO bulks and during their electromagnetic heating in operation, to accurately estimate the losses of energy and consumption of $\mathrm{LN}_{2}$, during these stages. Validation of the proposed models is performed comparing the evolutions of $\mathrm{LN}_{2}$ variables such as temperature, heat flux, gas concentration and mass loss, obtained from finite element method (FEM) simulation with the evolutions obtained from the experimental measurement.

Section II describes the coupled phenomena of YBCO/liquidnitrogen heat transfer and its liquid-gas phase change. Section III presents the finite element model developed on Comsol Multiphysics software. Validation of the model is shown in Sections IV to VI.

\section{Mathematical Formulation of the Problem}

The problem formulation is performed according to the physical phenomena that are verified on the different stages. During the initial ZFC stage, there is a phase change of $\mathrm{LN}_{2}$ from liquid to gas with the formation of gas bubbles, due to the 
TABLE I

VALUES AND CHARACTERISTICS CONSIDERED FOR $\rho, k$, AND $C_{p}$

\begin{tabular}{lccc}
\hline \hline Medium & $\rho\left(\mathrm{kg} \mathrm{m}^{-3}\right)$ & $k\left(\mathrm{~W} \mathrm{~m}^{-1} \mathrm{~K}^{-1}\right)$ & $C_{p}\left(\mathrm{~J} \mathrm{~kg}^{-1} \mathrm{~K}^{-1}\right)$ \\
\hline YBCO [8], [9] & 5900 & Eq. (3) & Eq. (4) \\
XPS foam [10] & 40 & 0.035 & 1300 \\
$\mathrm{LN}_{2}[11]$ & 808.5 & Eq. (5) & 2042 \\
\hline \hline
\end{tabular}

high-temperature difference in the boundary between $\mathrm{LN}_{2}$ and YBCO. A turbulent flow of bubbles with the formation of vortexes above the YBCO bulk surface occurs. During the operating stage, there is electromagnetic heating due to Joule losses from induced currents on YBCO bulk. Electromagnetic heating depends on the intensity and frequency of inducing electromagnetic fields. For magnetic fields with magnitudes and frequencies of the order of the ones experienced on the Superconducting Magnetic Bearing (SMB) in [4]-[6], the increase of the YBCO average temperature during the operation stage is gradual and phase change of $\mathrm{LN}_{2}$ from liquid to gas is done with no significant bubble releasing.

\section{A. Heat Transfer in the Different Medium}

The phenomena of heat transfer by convection-diffusion [7] can be traduced by equation (1).

$$
\rho C_{p} \frac{\partial T}{\partial t}+\rho C_{p} \boldsymbol{u} \cdot \nabla T-k \nabla^{2} T=Q
$$

where $T$ is the absolute temperature, $\boldsymbol{u}$ the velocity (that is null for the solid domains), $\rho$ the volumetric density, $k$ the heat conductivity and $Q$ the heating source volumetric power density. The specific heat capacity $C_{p}$ is the variation of enthalpy $H$ with temperature $T$ at constant pressure.

$$
C_{p}=\left(\frac{\partial H}{\partial T}\right)_{P} .
$$

Table I presents the values or characteristics considered for $\rho$, $k$ and $C_{p}$ in the solid YBCO bulk, the solid Extruded Polystyrene (XPS) foam and $\mathrm{LN}_{2}$ medium domains.

$$
\begin{aligned}
k_{\mathrm{YBCO}}= & 6.92+0.35 T+3.31 \times 10^{-3} T^{2}-1.68 \times 10^{-4} T^{3} \\
& +1 \times 10^{-6} T^{4} \\
C_{\mathrm{PYBCO}}= & 38.76+1.44 T \\
k_{\mathrm{LN} 2}= & 0.275-1.78 \times 10^{-3} \mathrm{~T} ; 63 \mathrm{~K} \leq T \leq 125 \mathrm{~K} .
\end{aligned}
$$

\section{B. Electromagnetic Heating by Induction}

The volumetric power density $Q$ due to electromagnetic heating from Joule losses is given by (6)

$$
Q=\boldsymbol{J} \cdot \boldsymbol{E}
$$

where $\boldsymbol{J}$ and $\boldsymbol{E}$ are the current density and electric field that are not null when the ZFC YBCO bulk is under the effect of changing magnetic fields [2], [3]. During the initial ZFC process, one may assume that $\boldsymbol{J}$ and $\boldsymbol{E}$ on the YBCO bulk and hence $Q$ are null because the cooling is done in the absence of any significant magnetic fields.

During operation, the zero-field-cooled YBCO is submitted to an alternating magnetic field $\boldsymbol{H}$, that induces a current in the superconductor. In our experiments, the inducing magnetic field $\boldsymbol{H}$ is generated by an alternating current source. For the quasi-stationary regime, the inducing magnetic field $\boldsymbol{H}$ relates with the source current density $\boldsymbol{J}_{\boldsymbol{s}}$ according to the Ampere's law in (7).

$$
\nabla \times \boldsymbol{H}=\boldsymbol{J}_{\boldsymbol{s}} .
$$

This can be derived in (8), where $\boldsymbol{A}$ is the magnetic potential vector and $\mu$ is the considered magnetic permeability of the medium.

$$
\nabla(\nabla \cdot \boldsymbol{A})-\nabla^{2} \boldsymbol{A}=\mu \boldsymbol{J}_{\boldsymbol{s}}
$$

Considering the Lorentz "gauge condition," this equation can be simplified to (9)

$$
\nabla^{2} \boldsymbol{A}=-\mu \boldsymbol{J}_{\boldsymbol{s}} .
$$

From the Faraday's law, the electric field $\boldsymbol{E}$ induced in the YBCO bulk, depends on the sourcing electric potential $V_{s}$ and on the magnetic potential vector $\boldsymbol{A}$ as in (10).

$$
\boldsymbol{E}=-\nabla V_{s}-\frac{\partial \boldsymbol{A}}{\partial t} .
$$

For the case that no sourcing electric potentials exist $\left(V_{s}=0\right)$, (10) can be approximated by (11) .

$$
\boldsymbol{E}=-\frac{\partial \boldsymbol{A}}{\partial t} .
$$

According to the model in [12], for the non-linear electric conductivity of YBCO, the current density $J$ relates with the induced electric field magnitude $E$ by (12).

$$
J=J_{C}\left(\frac{E}{E_{0}}\right)^{1 / n} .
$$

Here, $n$ is the non-linearity exponent equal to 21 and $E_{0}$ the electrical field for the critical current density $J_{C}$ depending on the magnetic flux density $B$ and on the absolute temperature $T$ by (13):

$$
J_{C}(B, T)=\alpha\left(1-\left(\frac{T}{T_{C}}\right)^{2}\right)^{3 / 2} \frac{B_{0}}{B_{0}+B} .
$$

Parameter $B_{0}$ is the magnetic flux density for which the critical current density assumes a value that is half the value with no magnetic field, $T_{C}$ is the critical temperature of superconductivity $93 \mathrm{~K}$ and $\alpha$ the critical current density value with no magnetic field, at absolute temperature zero. In the considered model, $E_{0}$ assumes a value equal to $10^{-4} \mathrm{~V} / \mathrm{m}, B_{0}$ a value equal $0.1 \mathrm{~T}$ and $\alpha$ a value equal to $1.134 \times 10^{8} \mathrm{~A} / \mathrm{m}^{2}$.

\section{Phase Change of Nitrogen From Liquid to Gas}

Whenever temperature surpasses $77 \mathrm{~K}$, there will be phase change from liquid to gas on the $\mathrm{LN}_{2}$ fluid domain. Equations 
(14) and (15) give, respectively, the effective values of volumetric density $\rho$ and of heat conductivity $k$ of liquid nitrogen to be considered in convection-diffusion equation (1).

$$
\begin{aligned}
& \rho=\theta \rho_{1}+(1-\theta) \rho_{2} \\
& k=\theta k_{1}+(1-\theta) k_{2} .
\end{aligned}
$$

Here, indexes 1 and 2 state for phase 1 (liquid) and phase 2 (gas), being $\theta$ the volumetric fraction of liquid in the mixture with gas. Variable $\theta$ assumes the unit before the phase change phenomena when no gas is mixed in $\mathrm{LN}_{2}$, a value between the unit and zero during the phase change from liquid to gas, and zero after the total change to the gas phase. An effective value of enthalpy $H$ given by (16) should be considered in (2).

$$
H=\frac{1}{\rho}\left(\theta \rho_{1} H_{1}+(1-\theta) \rho_{2} H_{2}\right) .
$$

Here, $H_{1}$ and $H_{2}$ are, respectively, the nitrogen enthalpies in phase 1 (liquid) and in phase 2 (gas) [7].

\section{Turbulent Flow Caused by the Releasing of Bubbles}

During the beginning of the YBCO ZFC process, a turbulent flow is generated around the superconductor surface by nitrogen gas released as bubbles. Such flow can be modeled by the NavierStokes general form equation for conservation of momentum [7] as

$$
\rho \frac{\partial \boldsymbol{u}}{\partial t}+\rho \boldsymbol{u} \cdot \nabla \boldsymbol{u}-\mu \nabla^{2} \boldsymbol{u}=-\nabla p
$$

being $\boldsymbol{u}$ the fluid velocity, $\rho$ the fluid volumetric density, $\mu$ the fluid dynamic viscosity and $p$ the pressure. In the case that the flow is performed with phase change, the effective fluid volumetric density changes and the continuity equation becomes

$$
\frac{\partial \rho}{\partial t}+\rho \nabla \cdot \boldsymbol{u}=m_{\mathrm{gl}}
$$

where $m_{\mathrm{gl}}$ is the mass density transfer rate from liquid to gas.

The Reynolds-Averaged Navier-Stokes (RANS) $k$-epsilon turbulence model [7] was adopted for simulation of the turbulent regime.

\section{E. Heat Transfer Between Different Medium}

Because of assumed homogeneity for all medium nearby boundaries, the continuity of the heat flux normal component was considered for all boundaries, as expressed in (19),

$$
\boldsymbol{q}_{m} \cdot \boldsymbol{n}_{m}=-\boldsymbol{q}_{n} \cdot \boldsymbol{n}_{n}
$$

where $\boldsymbol{q}_{\boldsymbol{m}} \cdot \boldsymbol{n}_{\boldsymbol{m}}$ is in medium $m$, the magnitude of the heat flux normal component to the boundary between mediums $m$ and $n$, that is pointing towards medium $m$.

Convective heat transfer according to Newton's convection law in (20) was considered, where the average heat flux $q_{e}$ at the boundary between $\mathrm{LN}_{2}$ and air is given by

$$
q_{e}=h\left(T_{\text {ext }}-T\right) .
$$

\begin{tabular}{|c|c|c|}
\hline Surface & $\boldsymbol{L}(\mathbf{m})$ & $\boldsymbol{h}\left(\mathbf{W} \mathbf{m}^{-2} \mathbf{K}^{-1}\right)$ \\
\hline Top and bottom horizontal plates & $0.031 \mathrm{~m}$ & 37 \\
\hline Vertical wall & $0.11 \mathrm{~m}$ & 8 \\
\hline
\end{tabular}

Here, $T$ is the temperature at the boundary, $T_{\text {ext }}$ the air temperature away from this boundary, and $h$ the heat transfer coefficient
TABLE II

VALUES of Heat TRansfer COEFFICIENTS By FREE CONVECTION ON HORIZONTAL SURFACE AND VERTICAL WALL

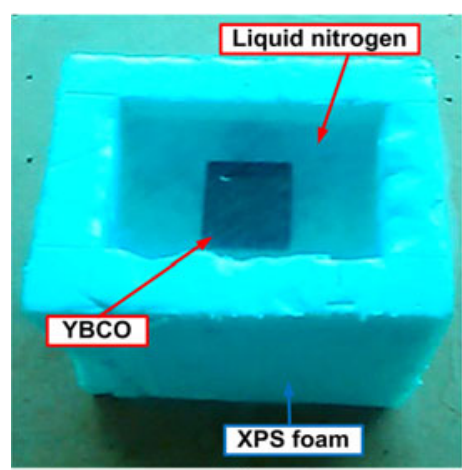

(a)

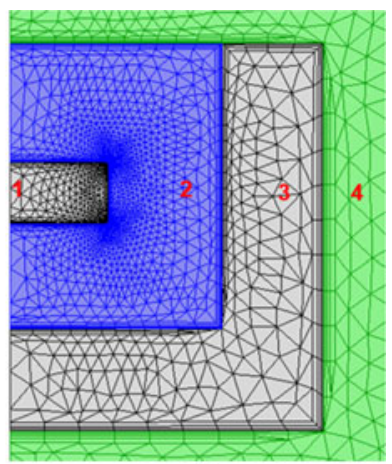

(b)
Fig. 1. (a) Rectangular box made of extruded polystyrene foam (Styrofoam) with a rectangular YBCO bulk submerged in $\mathrm{LN}_{2}$. (b) Axisymmetric geometry adopted on FEM.

considering heat transfer by free convection on environment air. Appropriate values for this coefficient for free convection on horizontal surfaces and vertical walls, can be estimated using the models given in [13], respectively by expressions (21) and (22).

$$
\begin{aligned}
& h_{h}=\frac{k}{L} 0.54 R_{\mathrm{aL}}^{1 / 4} \\
& h_{v}=\frac{k}{L} 0.68+\frac{0.67 R_{a L}{ }^{1 / 4}}{\left(1+\left(\frac{0.492 k}{\mu C_{p}}\right)^{9 / 16}\right)^{4 / 9}}
\end{aligned}
$$

where $L$ is a linear dimension given by the ratio between the horizontal area and perimeter or by the vertical wall high, $R_{a L}$ is the Rayleigh number with a value of $10^{7}$ to allow for heat transfer by convection in air, $k$ the thermal conductivity of air with a value of about $0.025 \mathrm{~W} \cdot \mathrm{m}^{-1} \cdot \mathrm{K}^{-1}, \mu$ the dynamic viscosity of air with a value of about $20 \times 10^{-6} \mathrm{~Pa} \cdot \mathrm{s}$ and $C_{p}$ the heat capacity of air with a value of about $1 \mathrm{~J} \cdot \mathrm{Kg}^{-1} \cdot \mathrm{K}^{-1}$. Table II shows the values assumed for $L$ basing on the box dimensions and calculated for the heat transfer coefficients by free convection on the horizontal surfaces $h_{h}$ and vertical wall $h_{v}$.

\section{Finite ELEMENT Modeling}

The real system tested represented in Fig. 1(a), consists on a rectangular box with $20 \mathrm{~mm}$ thick walls made of XPS material, having the above surface totally open to the air, and a rectangular single-seed YBCO bulk from CAN SUPERCONDUCTORS with dimensions $12 \times 25 \times 25 \mathrm{~m}$. Table III lists the main characteristic values of the YBCO. The bulk stands on the top of four thin rods made of steel, each rod with $3 \mathrm{~mm}$ diameter, submerged in the middle of the $\mathrm{LN}_{2}$ medium filling the box rectangular cavity with dimensions $55 \times 60 \times 90 \mathrm{~m}$. The distance between 
TABLE III

YBCO CHARACTERISTIC VALUES

\begin{tabular}{ccc}
\hline \hline Material & Parameter & Value \\
\hline & $n$ & 20 \\
YBCO & $J_{C 0}$ & $1.82 \times 10^{8} \mathrm{~A} / \mathrm{m}^{2}$ \\
& $B_{0}$ & $0.1 \mathrm{~T}$ \\
\hline \hline
\end{tabular}

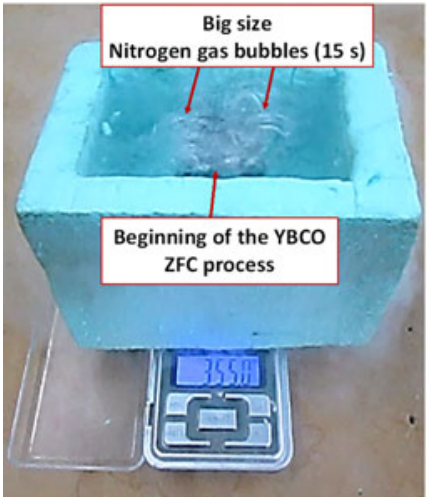

(a)

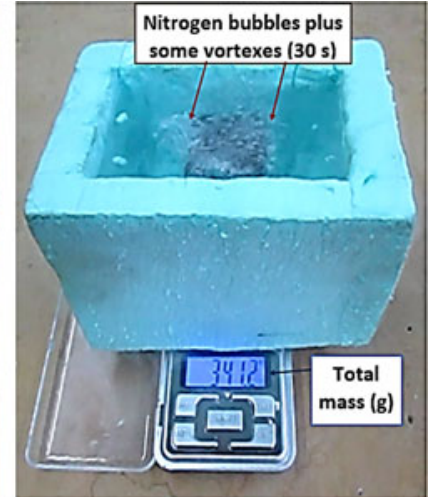

(b)
Fig. 2. Verified ZFC thermodynamic phenomena: a) $15 \mathrm{~s}$ and b) $30 \mathrm{~s}$ after immersing the YBCO bulk in $\mathrm{LN}_{2}$.

the box cavity bottom and the bulk inferior surface was about $20 \mathrm{~mm}$.

Fig. 1(b) shows the transversal section view of the axisymmetric geometry adopted on Finite Element (FE) modeling. The volumes of the YBCO bulk and of $\mathrm{LN}_{2}$, and the total area of contact between the $\mathrm{LN}_{2}$ and the YBCO bulk or the box walls, all kept on the axisymmetric geometry.

Regions 1, 2, 3 and 4 correspond, respectively, to the YBCO bulk, the $\mathrm{LN}_{2}$, the box walls and the environment air domains. An unstructured hybrid mesh configuration was adopted, with tetrahedral elements nearby the boundaries, thus having longer and shorter sides, respectively, parallel and perpendicular to the boundaries to account for higher temperature and pressure gradients in these regions. The other elements conform to Delaunay's tessellation scheme.

\section{ZERO-FIELD COOLING}

This section evaluates and presents results about the thermodynamic processes in $\mathrm{LN}_{2}$ during the initial zero-field-cooling process of a YBCO from the initial environment temperature of $20{ }^{\circ} \mathrm{C}(293.15 \mathrm{~K})$ down to the YBCO critical temperature of superconductivity $T_{c}(93 \mathrm{~K})$.

As observed in practice, after immersing the YBCO bulk in $\mathrm{LN}_{2}$, there is phase change with the formation of big sized nitrogen gas bubbles nearby the boundaries between YBCO and $\mathrm{LN}_{2}$, as indicated in Fig. 2(a) and (b). Due to releasing of bubbles, a turbulent flow of LN2 occurs in the box cavity, as shown in Fig. 3(a) for the first 15 s, and Fig. 3(b) at 30 seconds, respectively. Also, vortexes are generated and caused by the upward flow of $\mathrm{LN}_{2}$ that occurs nearby the bubbles releasing

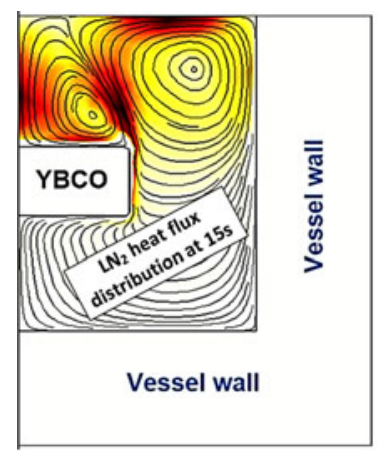

(a)

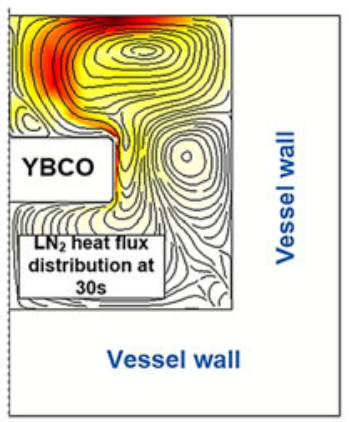

(b)
$\left[\mathrm{W} / \mathrm{m}^{2}\right]$

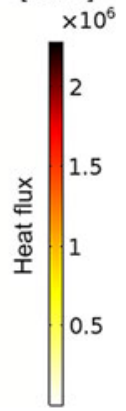

Fig. 3. Heat flux distribution $\left(\mathrm{W} / \mathrm{m}^{2}\right)$ in $\mathrm{LN}_{2}$ and heat stream contours at a) $15 \mathrm{~s}$ and b) $30 \mathrm{~s}$. Vortices appear mainly above the superconductor due to the strong upward velocity of the nitrogen bubbles that were formed.

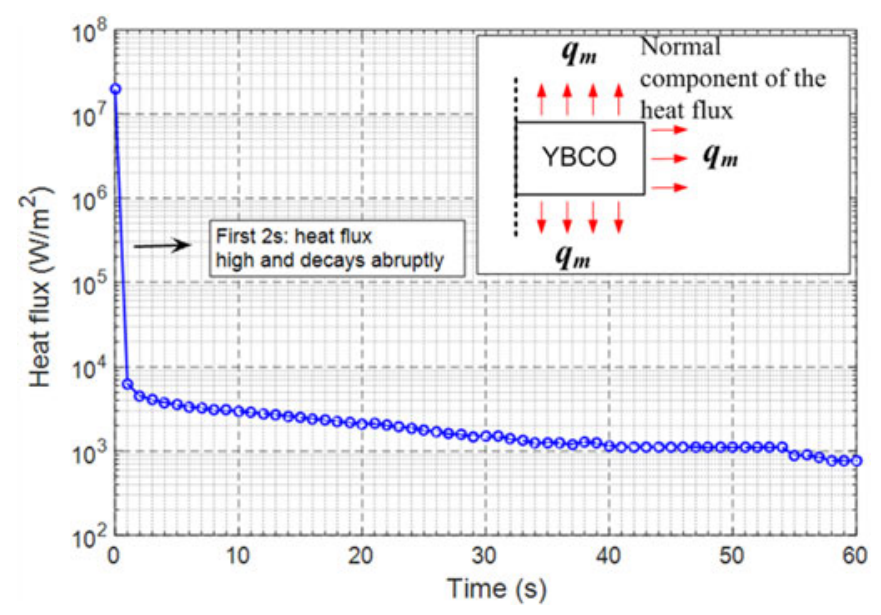

Fig. 4. FE model. Evolution of the average heat flux normal to the boundary between $\mathrm{YBCO}$ bulk and $\mathrm{LN}_{2}$.

zone and also generated by the downward flow of $\mathrm{LN}_{2}$ nearby the box walls.

Figs. 3(a) and (b) show the heat flux distribution in $\mathrm{LN}_{2}$ and its flux stream contours in 15 and 30 seconds after the beginning of the cooling process. These results show that during the first 30 seconds, one can notice that the upward velocity of the nitrogen bubbles is such that there is the formation of vortices just above the YBCO top surface. Approximately after this period, vortices tend to disappear with the gradual size reduction and velocity of bubbles due to a continuing decrease of heat flux magnitude.

Fig. 4 presents the evolution of $\boldsymbol{q}_{\boldsymbol{m}}$, the normal component of the heat flux in the YBCO- $\mathrm{LN}_{2}$ boundary surface. During the first 2 seconds, heat flux leaving out of the YBCO to $\mathrm{LN}_{2}$ is very high and decays abruptly. After, the heat flux exchange is much less intense, decaying gradually at an almost constant rate.

Fig. 5 shows how the average temperature of the $\mathrm{YBCO}$ bulk changes during 60 seconds according to the FE model prediction. The model predicts that the necessary time to zero field cool the YBCO bulk down to its critical temperature (93 K) will stay about 1 minute.

Fig. 6 shows the plot of the temperature distribution and corresponding isothermal contours at 60 seconds after the 


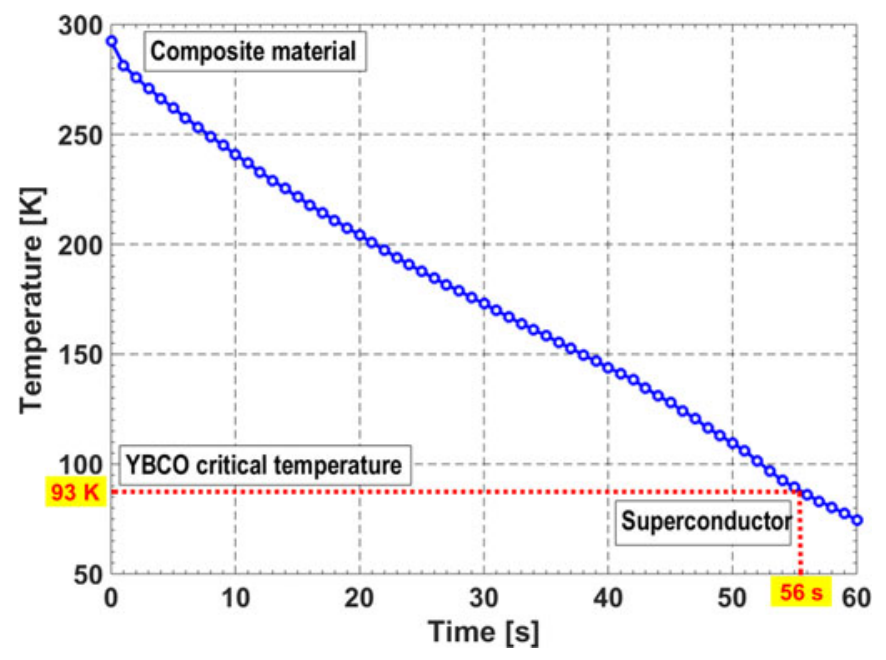

Fig. 5. Predicted evolution of the YBCO block average temperature by FE simulation.

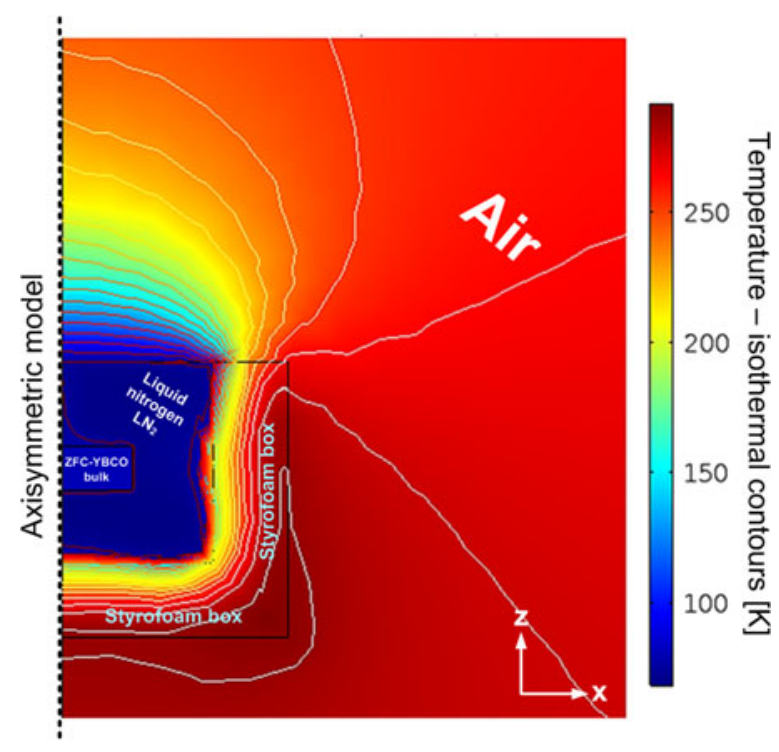

Fig. 6. Numerical simulation results for the isothermal contour map of the temperature distribution in the ZFC-YBCO bulk, Styrofoam box at $60 \mathrm{~s}$ after the beginning of the cooling process.

beginning of the ZFC process. The result shows that, at this time, the superconductor has a temperature equal to that of the liquid nitrogen.

Fig. 7 compares the rate of loss of $\mathrm{LN}_{2}$ mass during phase change, obtained from FE simulation with the one experimentally measured using the digital balance shown in Fig. 2 with a resolution of $100 \mathrm{mg}$. From Fig. 7, the quantity of $\mathrm{LN}_{2}$ lost during the first $42 \mathrm{~s}$ of the ZFC process is previewed to be about $-12 \%$. Doing the experiment, a loss of $-14 \%$ was achieved in reality as indicated in Fig. 7, which shows the very accurate prevision of the model.

\section{HEATING OF THE ZFC-YBCO BY THE INFLUENCE OF ROOM TEMPERATURE}

This section presents results about the loss of $\mathrm{LN}_{2}$ mass due to phase change by the influence of room temperature, after the

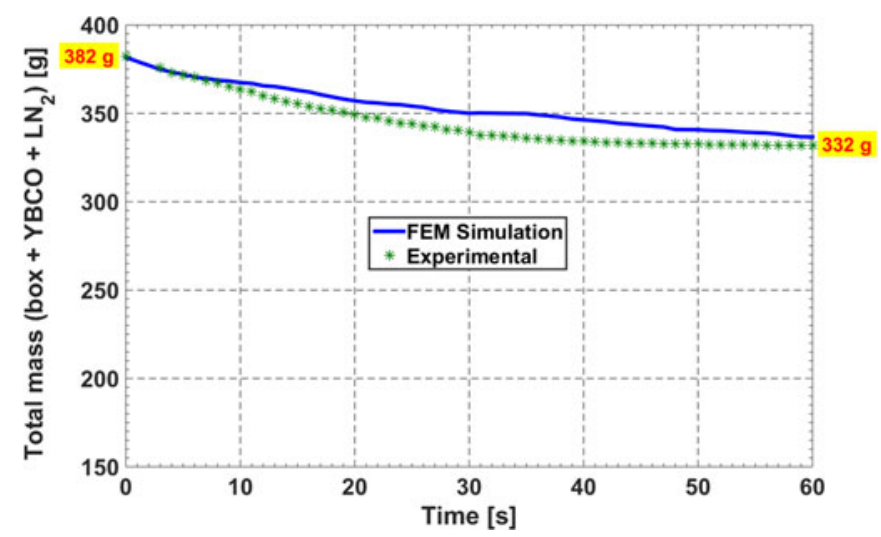

Fig. 7. Numerical simulation and experimental results for the evolution of total mass (box $+\mathrm{LN}_{2}+\mathrm{YBCO}$ ). The $\mathrm{LN}_{2}$ lost during $\mathrm{ZFC}$ is previewed to be about $-12 \%$. Doing the experiment, a loss of $-14 \%$ was achieved.

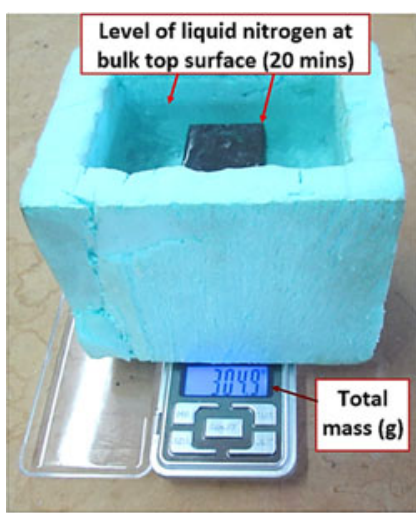

(a)

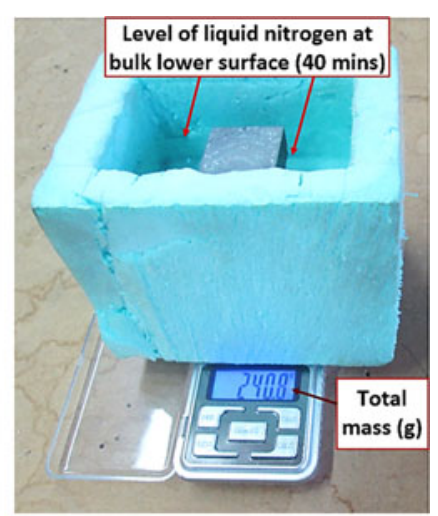

(b)
Fig. 8. Total mass measured at instants a) $20 \mathrm{~min}$ and b) $40 \mathrm{~min}$ when the level of $\mathrm{LN}_{2}$ reaches the top and lower superconductor surfaces, respectively.

ZFC of YBCO bulks. On the analysis, it was assumed that the box is previously fulfilled with $\mathrm{LN}_{2}$ and that the initial average temperature of the YBCO bulk is $70 \mathrm{~K}$. A room temperature about $20{ }^{\circ} \mathrm{C}(293.15 \mathrm{~K})$ was measured and considered on predictions with FEM simulation. If no pouring of $\mathrm{LN}_{2}$ is guaranteed, the level of $\mathrm{LN}_{2}$ in the box decreases progressively due to a phase change of nitrogen from liquid to gas by the influence of the room temperature. It was verified that the $\mathrm{LN}_{2}$ level reached the top and lower superconductor surfaces, respectively, after about 20 min and 40 min, as shown in Fig. 8(a) and (b), respectively. These show the total measured mass at these two-time instants: from $382 \mathrm{~g}$ to $304.9 \mathrm{~g}$ after $20 \mathrm{~min}$, and from $304.9 \mathrm{~g}$ to $240.8 \mathrm{~g}$ after more $20 \mathrm{~min}$.

With the superconductor totally immersed in $\mathrm{LN}_{2}$, the release of bubbles was not notable, being the $\mathrm{LN}_{2}$ flow velocities much lower than during the initial ZFC process. Due to this reason, a laminar flow physics was considered in modeling the $\mathrm{LN}_{2}$ when the heating superconductor by the influence of room temperature. The heat transfer by free convection physics was considered for the XPS walls in contact with air, using the heat transfer coefficients listed before in Table II. 


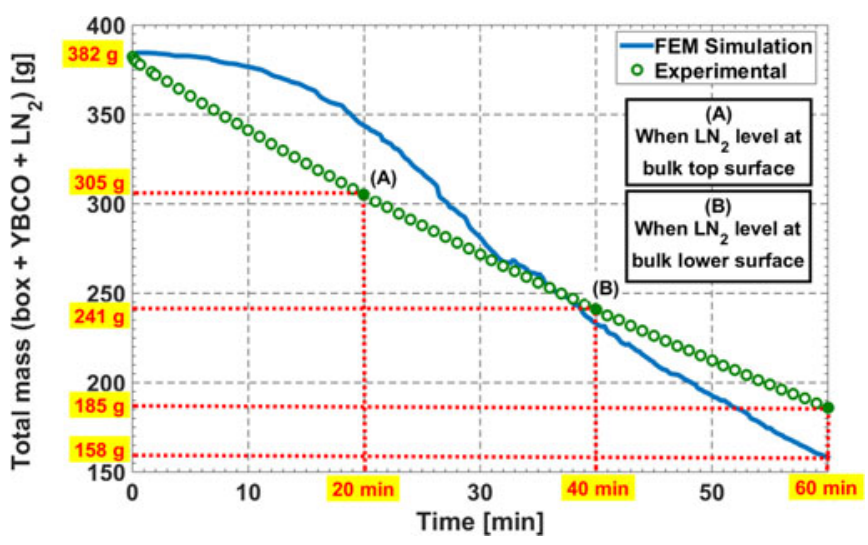

Fig. 9. Numerical simulation and experimental results for the evolution of total mass (box $+\mathrm{LN}_{2}+\mathrm{YBCO}$ ).

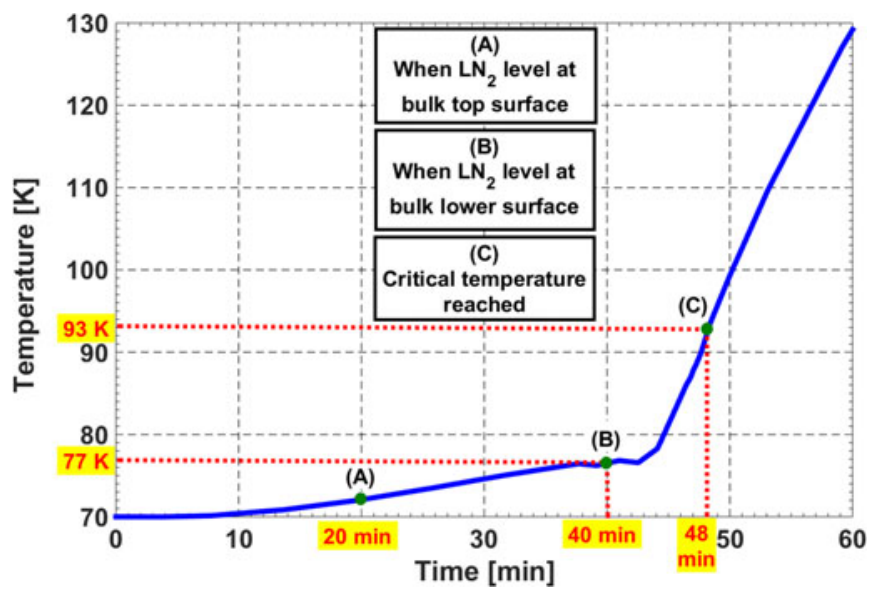

Fig. 10. Numerical simulation results for the average temperature of the YBCO bulk.

Fig. 9 shows the evolution of total $\mathrm{LN}_{2}$ mass, both measured in the lab with the digital balance and that predicted by the FE model (continuous line). The measured average rate of $\mathrm{LN}_{2}$ consumption during the heating was about $3.3 \mathrm{~g} / \mathrm{min}$, while the model predicted an $\mathrm{LN}_{2}$ mass consumption rate of about $3.7 \mathrm{~g} / \mathrm{min}$, thus showing the very accurate precision. The measured weight of the four rods plus the bulk and the cooled box was $150 \mathrm{~g}$. It was also verified that the box becomes almost empty of $\mathrm{LN}_{2}$ after one hour. This can be confirmed subtracting this weight from the total mass.

Fig. 10 shows the predicted evolution of the YBCO bulk average temperature obtained by FEM simulation. From this, one may conclude that the superconductor average temperature remains lower than the boiling temperature of $\mathrm{LN}_{2}$ whenever the bulk remains immersed. This was confirmed experimentally because no bubbles were formed on the boundary surfaces between the YBCO and $\mathrm{LN}_{2}$ during the time the $\mathrm{YBCO}$ was immersed. Results in Fig. 10 also show that the YBCO average temperature increases more rapidly when the $\mathrm{LN}_{2}$ level is below the bulk lower surface being totally in contact with the nitrogen gas. Before, one can estimate an increasing temperature rate of $+0.23 \mathrm{~K} / \mathrm{min}$, while after the rate almost increase ten times to about $+3.33 \mathrm{~K} / \mathrm{min}$.

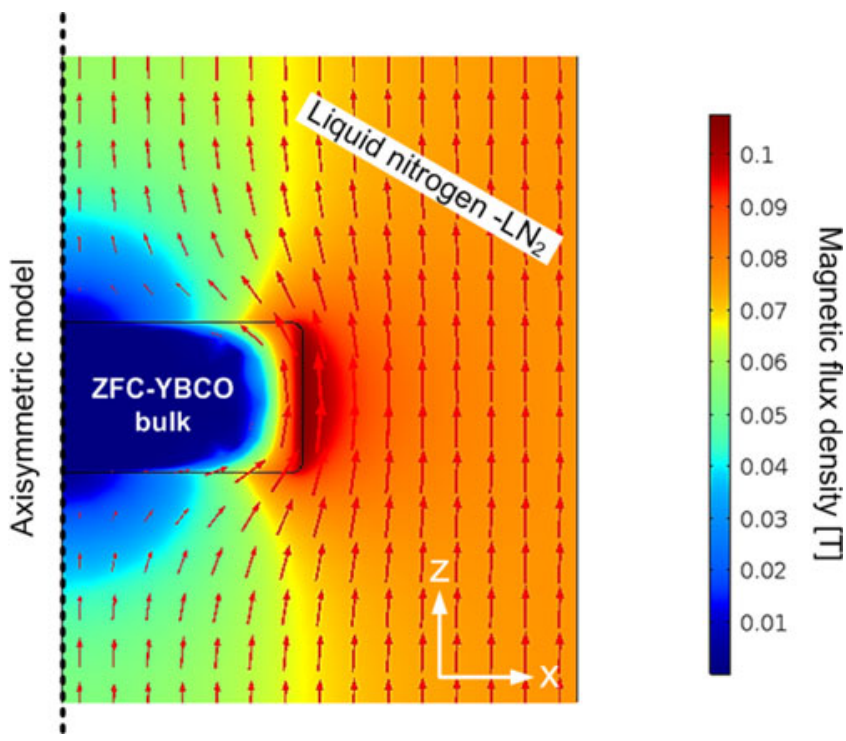

Fig. 11. Numerical simulation results of the magnetic flux density when subjected to a sinusoidal magnetic flux density $\boldsymbol{B}$ having $0.25 \mathrm{~T}$ amplitude and $2 \mathrm{~Hz}$. Results from a ZFC YBCO condition.

\section{HEATING OF THE ZFC-YBCO By EleCtromagnetiC INDUCTION}

This section evaluates the increase of the YBCO bulk temperature by electromagnetic induction when submitted to a magnetic field with amplitude and frequency similar to the one experienced by the superconducting magnetic bearing (SMB) prototype described in [4]-[6]. Electromagnetic heating of the YBCO by the induced currents can cause the appearing of bubbles on the boundary between the $\mathrm{YBCO}$ and $\mathrm{LN}_{2}$, whenever the YBCO surface temperature becomes higher than the $\mathrm{LN}_{2}$ boiling temperature $(77 \mathrm{~K})$. This effect can appear in the SMB due to the $\mathrm{NdFeB}$ permanent magnets (PMs) used on its rotor, which is very close to their surface and in the main PMs polarization direction, making the YBCO subject to an almost B field with a magnitude of about $0.25 \mathrm{~T}$.

In this study, is assumed that the ZFC YBCO bulk stands immersed in $\mathrm{LN}_{2}$, being subjected to a uniformly distributed sinusoidal magnetic induction field with amplitude $0.25 \mathrm{~T}$ and frequency $2 \mathrm{~Hz}$. The heating by the influence of room temperature is null. The initial temperature of $\mathrm{LN}_{2}$ is $70 \mathrm{~K}$. Heat transmission in the nitrogen has been due to conduction and thermal convection. Hence, as the rate of temperature rise was low, the simulation is expected to take 28 seconds to reach $77 \mathrm{~K}$ at the maximum temperature points on the surface of the superconductors. With this condition, the thermal conductivity of the nitrogen is not affected by the liquid nitrogen percentage value, and thus transmission heat with phase change dynamics has not been included in the FE model. As the $77 \mathrm{~K}$ is not reached, there is also no bubble formation and the speed of $\mathrm{LN}_{2}$ will depend only on changes in temperature, not bubble formation.

Fig. 11 shows for a certain time instant the spatial distribution of field $\boldsymbol{B}$ inside and outside of the superconductor. Fig. 12 shows the evolution of the average current density induced on the YBCO during 4 cycles. For these four cycles, Fig. 13 shows the 


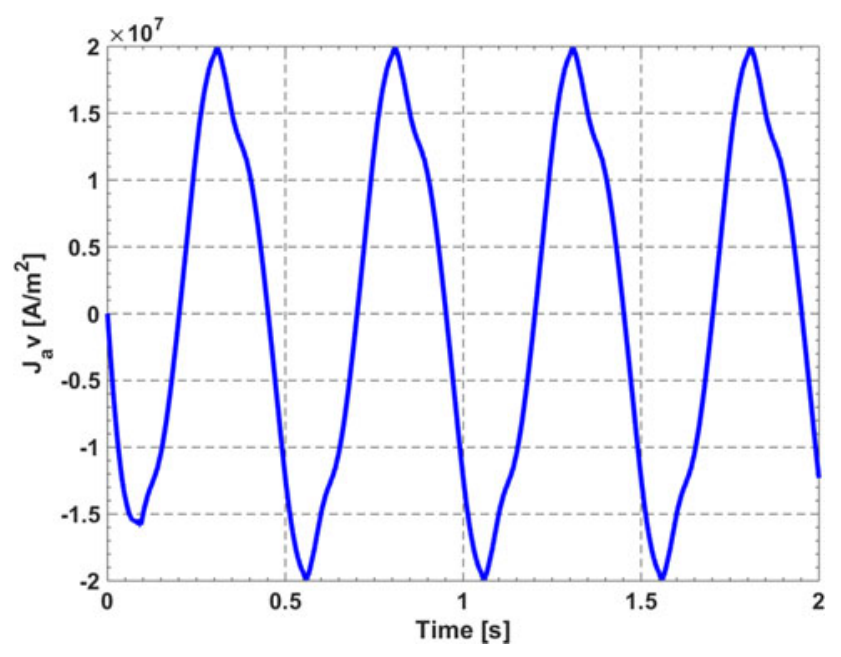

Fig. 12. Numerical simulation results of time evolution of the average current density across the interior of the YBCO bulk, during four cycles with a frequency of $2 \mathrm{~Hz}$.

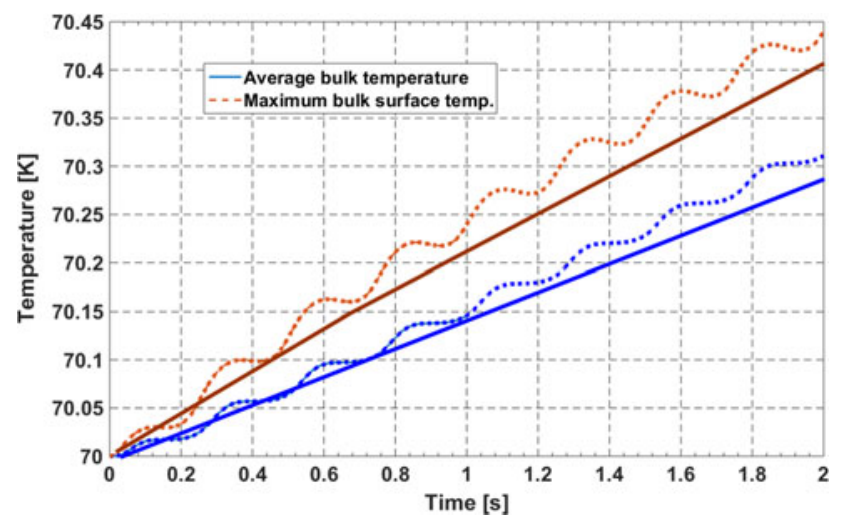

Fig. 13. Numerical simulation results of the time evolution of the average bulk temperature (blue dotted) and of the maximum temperature value at the boundary surface between $\mathrm{YBCO}$ and $\mathrm{LN}_{2}$ domains.

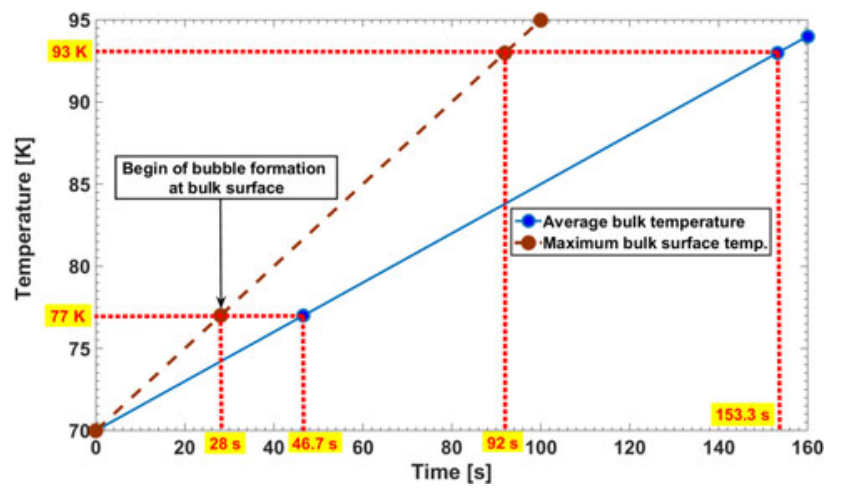

Fig. 14. Prediction of temperature evolution tendency for a wider time range.

predicted evolution of the average bulk temperature (blue color) and also of the maximum surface temperature (brown color) at the boundary between $\mathrm{YBCO}$ and $\mathrm{LN}_{2}$. The predicted rate of increase of the bulk average temperature was about $0.15 \mathrm{~K} / \mathrm{s}$. The predicted rate of increase of the maximum bulk surface temperature at the boundary between $\mathrm{YBCO}$ and $\mathrm{LN}_{2}$ domains was almost two times to $0.25 \mathrm{~K} / \mathrm{s}$.
Fig. 14 represents the extension of the two straight lines traced in Fig. 13, in time, until the YBCO critical temperature of superconductivity $(93 \mathrm{~K})$ is reached. As seen, the maximum bulk surface temperature could increase by $7 \mathrm{~K}$ and some bubbling could appear at the boundary between the $\mathrm{YBCO}$ and $\mathrm{LN}_{2}$ domains after about $28 \mathrm{~s}$. Another important conclusion is that the superconductivity could start to be initially lost at the YBCO bulk surface after about $92 \mathrm{~s}$.

\section{CONCLUSION}

This paper evaluated and validated models for finite element analysis of the thermodynamic processes, during the initial zerofield cooling of a YBCO bulk and under its electromagnetic inductive heating due to an alternate magnetic flux density. The results have shown that, when a YBCO bulk at room temperature is suddenly submerged in $\mathrm{LN}_{2}$, the necessary time to zerofield cool the YBCO down to its critical temperature is about 1 minute. Due to the high-temperature differences, the heat transfer rate is very high during the first 2 seconds. After this initial transient, heat transfer rate becomes less intense decaying gradually until the YBCO reaches the critical temperature of superconductivity. During this process, there is a phase change of $\mathrm{LN}_{2}$ from liquid to gas with notable releasing bubbles. The measured consumption of $\mathrm{LN}_{2}$ during the initial ZFC process was about $14 \%$. Similarly, the $\mathrm{LN}_{2}$ mass consumption that was predicted by FEM simulation was about $12 \%$, which indicated the very accurate precision of the developed fluid flow and heat transference with phase-change multiphysics model.

The average temperature of the ZFC YBCO bulk is lower than the $\mathrm{LN}_{2}$ boiling temperature whenever the YBCO bulk remains totally immersed in $\mathrm{LN}_{2}$ and not under influence of any significant electromagnetic induction. This was verified experimentally because, under these conditions, no gas bubbles are formed on the boundary between $\mathrm{YBCO}$ and $\mathrm{LN}_{2}$ elements. Experiments presented an average rate of $\mathrm{LN}_{2}$ consumption around $3.3 \mathrm{~g} / \mathrm{min}$ during the YBCO heating caused only by the influence of room temperature. The predicted of mass loss rate by FEM simulation was close to this value, giving $3.7 \mathrm{~g} / \mathrm{min}$, showing again the very accurate precision of the FE model.

Electromagnetic heating by induced currents in the superconductor makes bubbles of nitrogen gas appear on the boundary between the $\mathrm{YBCO}$ and $\mathrm{LN}_{2}$ whenever the $\mathrm{YBCO}$ surface temperature becomes higher than the $\mathrm{LN}_{2}$ boiling temperature $(77 \mathrm{~K})$. Simulation results have shown that on the heating by only electromagnetic induction, under a magnetic field with magnitude $0.25 \mathrm{~T}$ and frequency $2 \mathrm{~Hz}$, some bubbling could appear at the boundary between the $\mathrm{YBCO}$ and $\mathrm{LN}_{2}$ domains after about $28 \mathrm{~s}$ and superconductivity could start to be lost at YBCO surface after about $92 \mathrm{~s}$.

\section{REFERENCES}

[1] P. J. C. Branco and J. A. Dente, "Design and experiment of a new maglev design using zero-field-cooled YBCO superconductors." IEEE Trans. Ind. Electron., vol. 59, no. 11, pp. 4120-4127, Nov. 2012.

[2] J. Fernandes, I. Montes, R. Sousa, C. Cardeira, and P. J. C. Branco, "Superconductor joule losses in a zero-field-cooled (ZFC) maglev vehicle," IEEE Trans. Appl. Supercond., vol. 26, no. 3, Apr. 2016, Art. no. 8201907. 
[3] J. Arnaud and P. J. C. Branco, "Electrothermal characteristics of YBCO bulk magnets deep in LN2: A preliminary analysis for its use as excitation system of low-speed synchronous generators," IEEE Trans. Appl. Supercond., vol. 26, no. 3, April 2016, Art. no. 6800608.

[4] A. J. Arsénio, M. V. Carvalho, C. Cardeira, R. Melício, and P. J. C Branco, "Experimental setup and efficiency evaluation of zero-field-cooled (ZFC) YBCO magnetic bearings," IEEE Trans. Appl. Supercond., vol. 27, no. 4, Jun. 2017, Art. no. 3601105.

[5] A. J. Arsénio, M. V. Carvalho, C. Cardeira, R. Melício, and P. J. C Branco, "Conception of a YBCO superconducting zfc-magnetic bearing virtual prototype," in Proc. IEEE Int. Power Electron. Motion Control Conf. (PEMC), Bulgaria, 25-28 Sept. 2016, pp. 1226-1230.

[6] A. J. Arsénio, M. V. Carvalho, C. Cardeira, R. Melício, and P. J. C Branco, "Viability of a frictionless bearing with permanent magnets and HTS bulks," in Proc. IEEE Int. Power Electron. Motion Control Conf. (PEMC), Bulgaria, 25-28 Sept. 2016, pp. 1231-1236.

[7] R. W. Lewis, P. Nithiarasu, and K. N. Seetharamu, Fundamentals of the Finite Element Method for Heat and Fluid Flow. Hoboken, NJ: Wiley, 2004.
[8] M. Zhang, K. Matsuda, and T. A. Combs, "New application of temperature-dependent modeling of high-temperature superconductors: Quench propagation and pulse magnetization,' J. Appl. Phys., vol. 112, 2012, Art. no. 043912.

[9] J. Feng, "Thermohydraulic-quenching simulation for superconducting magnets made of YBCO HTS tape," in Plasma Science and Fusion Center - Massachusetts Institute of Technology. Cambridge, Tech. Rep., 2110.

[10] E. Placido, M. C. Arduini-Schuster, and J. Kuhn, "Thermal properties predictive model for insulating foams," Infrared Phys. Technol., vol. 46, pp. 219-231, 2005

[11] E. W. Lemmon and R. T. Jacobsen, "Viscosity and thermal conductivity equations for nitrogen, oxygen, argon and air," Int. J. Thermophysics, vol. 25, no. 1, pp. 21-69, Jan. 2004.

[12] Z. Hong, H. M. Campbell, and T. A. Coombs, "Computer modeling of magnetization in high-temperature superconductors," IEEE Trans. Appl. Supercond., vol. 17, no. 2, pp. 3761-3764, 2007.

[13] F. P. Incropera, D. P. Dewit, T. L. Bergman, and A. S. Lavine, Fundamentals of Heat and Mass Transfer, 6th ed. Hoboken, NJ: Willey, Copyright, 2007. 